\title{
Tests against noncontextual models with measurement disturbances
}

\author{
Jochen Szangolies,,${ }^{1,2, *}$ Matthias Kleinmann, ${ }^{2}, \dagger$ and Otfried Gühne ${ }^{2, \ddagger}$ \\ ${ }^{1}$ Institut für Theoretische Physik III, Heinrich-Heine-Universität Düsseldorf, D-40225 Düsseldorf, Germany \\ ${ }^{2}$ Naturwissenschaftlich-Technische Fakultät, Universität Siegen, \\ Walter-Flex-Straße 3, D-57068 Siegen, Germany
}

\begin{abstract}
The testability of the Kochen-Specker theorem is a subject of ongoing controversy. A central issue is that experimental implementations relying on sequential measurements cannot achieve perfect compatibility between the measurements and that therefore the notion of noncontextuality does not apply. We demonstrate by an explicit model that such compatibility violations may yield a violation of noncontextuality inequalities, even if we assume that the incompatibilities merely originate from context-independent noise. We show, however, that this problem can be circumvented by combining the ideas behind Leggett-Garg inequalities with those of the Kochen-Specker theorem.
\end{abstract}

PACS numbers: 03.65.Ta, 03.65.Ud

\section{INTRODUCTION}

Bell's theorem [1] is a famous no-go result that provides constraints on the program of interpreting quantum mechanics as an incomplete theory in the sense of Einstein, Podolsky, and Rosen [2]. It is expressed via inequalities that are fulfilled by any local realistic theory, but which are predicted to be violated by quantum mechanics. Experimentally, it is indeed found that quantum mechanics violates these inequalities for certain entangled states [3, 4]. Similar to Bell, Leggett and Garg [5] have proposed inequalities that are fulfilled by theories that satisfy a criterion of macroscopic realism, meaning that a system always occupies one of the states accessible to it. Under the further assumption of measurement non-invasiveness, the correlations between measurements performed on the system at different points in time obey a bound that is violated by quantum mechanics.

A third no-go result is provided by the Kochen-Specker theorem [6, 7]. Essentially, it replaces Bell's assumption of locality with the condition of noncontextuality: the outcome of a measurement on a system should not depend on other compatible measurements performed on the same system. Here, two measurements are called compatible, if they can be measured simultaneously or in a temporal sequence without any disturbance. The Kochen Specker result contains Bell's theorem as a special case in which the measurements are performed at spatial separation [8]. It is, however, also applicable to single quantum systems; consequently, entanglement is not necessary for violations of noncontextuality. In fact, violations of Kochen-Specker inequalities occur for all quantum systems of dimension $d \geq 3$, independent of the initial quantum state [6].

However, in contrast to Bell's theorem, the KochenSpecker theorem does not readily lend itself to experi-

\footnotetext{
*Electronic address: szangolies@thphy.uni-duesseldorf.de

${ }^{\dagger}$ Electronic address: matthias.kleinmann@uni-siegen.de

‡Electronic address: otfried.guehne@uni-siegen.de
}

mental tests of quantum mechanics. The direct testability is stymied by the fact that, during each experiment, only one measurement context is accessible at any given time. This limitation can be overcome, however, because similarly to Bell's theorem, the Kochen-Specker theorem can be expressed using inequalities, though it was originally not cast into this form $[9,10]$. This permits testing quantum contextuality by using measurements that are carried out sequentially.

But even in this formulation, the question of experimental testability poses further difficulties. The reason for this lies in the notion of contextuality, which only applies in the case of compatible observables. But in an experiment, this condition cannot be fulfilled perfectly; indeed, even measuring the same observable twice may yield different results. This is due to the unavoidable presence of noise during the measurement process, which leads to disturbances of the state. It has been argued that this inherent difficulty, together with a related issue concerning the finite precision of real measurements, nullifies the physical significance of the Kochen-Specker theorem [11-13].

Different strategies have been proposed to overcome this problem. In Ref. [13], the modification of KochenSpecker inequalities through the introduction of error terms was considered (see also Ref. [14]). Given that the measurement-induced disturbances are cumulative, these terms compensate for the violations of compatibility. A related approach, addressing a similar loophole in experimental tests of Leggett-Garg inequalities, was proposed in Ref. [15]. On the other hand it was suggested in Ref. [16] to perform experiments on separate qutrits in order to forestall the possibility of violations of compatibility.

In this paper, we take a different approach. First, we consider the question: what does an experimentally observed violation of a noncontextuality inequality license us to conclude? By proposing an explicit model capturing the effects of noise-induced compatibility violations, we argue that to conclude contextuality from the violation alone is difficult to justify: the model produces violations of noncontextuality inequalities while being in- 
dependent of the measurement context, and thus, noncontextual in this sense. In particular, we show that even the introduction of error terms as proposed in Ref. [13] cannot settle the issue.

We then propose a way to circumvent this problem by taking into account the ideas of Leggett and Garg: imposing a suitable time-ordering onto the measurements, it turns out to be possible to formulate inequalities that cannot be violated within our framework, and thus, allow to rule out more general hidden-variable models under realistic experimental conditions.

This paper is organized as follows. In Section II, we briefly recall the notions of compatibility and contextuality. Then, we propose an explicit classical noise model capable of inducing compatibility violation in such a way as to violate contextuality inequalities. In Section III we show that the model cannot be ruled out by previous approaches. To remedy this problem, in Section IV we propose new inequalities, utilizing ideas from Leggett and Garg. These inequalities allow to rule out more general hidden variable models.

\section{NONCONTEXTUAL MODELS}

As a basis for our investigations we take a variant of the well-known Clauser-Horne-Shimony-Holt (CHSH) inequality [17]

$$
\left\langle\chi_{\mathrm{CHSH}}\right\rangle=\langle A B\rangle+\langle B C\rangle+\langle C D\rangle-\langle D A\rangle \stackrel{N C H V}{\leq} 2 \stackrel{Q M}{\leq} 2 \sqrt{2} .
$$

Each of the observables $A, B, C, D$ has outcomes \pm 1 , and $\langle A B\rangle$ denotes the average over many repetitions obtained by measuring first $A$, then $B$, and then multiplying the results. If we assume that the observables in each context $\langle A B\rangle$, etc., are compatible, then $N C H V$ denotes the classical (noncontextual hidden-variable) bound, i.e., the value obtained if each of the observables is assumed to have a fixed value independently of which context it is measured in. The bound $Q M$ denotes the maximal value achievable in quantum mechanics [18]. The question now is: suppose one experimentally observes $\left\langle\chi_{\mathrm{CHSH}}\right\rangle>2$. Is this sufficient to conclude contextual behavior?

First, we need to make the notions of compatibility and noncontextuality precise. Consider some observables $\mathcal{O}=\{A, B, C, \ldots\}$. Compatibility then means that within any sequence of measurements composed of these observables, the observed value does not depend on the point at which it is measured within the sequence. That is, for any sequence of compatible measurements $\mathcal{C}$, the observed value of $O$ at the $i$ th point in the sequence, $v_{i}(O \mid \mathcal{C})$, does not depend on $i$, i.e., $v_{i}(O \mid \mathcal{C})=v_{j}(O \mid \mathcal{C})$ for all $i$ and $j$. This formalizes the notion that measurement of one observable does not influence the measurement of any other observable.

Then, any set of compatible observables $\mathcal{C}$ is called a context. A theory is called noncontextual, if for all observables $O$ and for all contexts $\mathcal{C}, \mathcal{C}^{\prime}$ the observed value

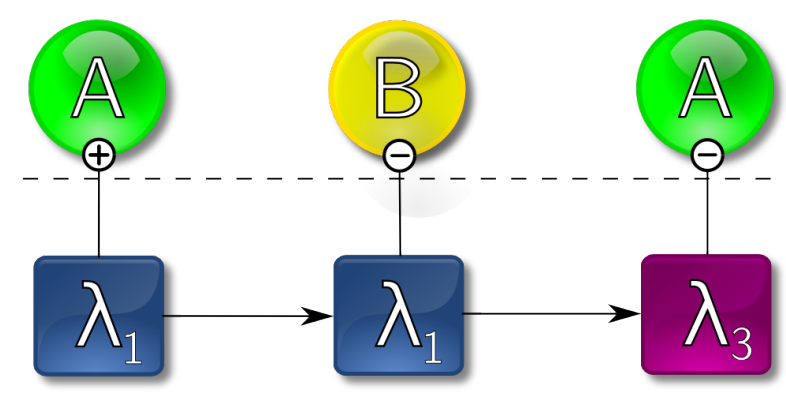

FIG. 1: Schematic representation of a sequence of measurements. Measurements $A$ and $B$ are performed sequentially on a system whose (hidden-variable) state $\lambda$ evolves stochastically as indicated. Time runs left to right.

is independent of the context, i.e., $v(O \mid \mathcal{C})=v\left(O \mid \mathcal{C}^{\prime}\right)$. Note that through the definition of a context, the notion of noncontextuality explicitly depends on compatibility.

To approach the question, we construct a counterexample given by a simple model for noise-induced disturbances of the hidden-variable states. These hidden states $\lambda_{i}$ are assumed to completely specify all possible experimental outcomes. In the present case, they can thus be indexed by the dichotomic outcomes of measurements of the observables $\mathcal{O}=\{A, B, C, D\}$ : a given state is specified uniquely by a set of values $v(O) \in\{ \pm 1\}$ for all $O \in \mathcal{O}$. For ease of notation, this set of values may be interpreted as a binary number, whose decimal value is used to index the state, i.e., $\lambda_{2}=\lambda_{(++-+)}$denotes the state that produces the measurement results $A=+1$, $B=+1, C=-1$ and $D=+1$. The model can be generalized by considering states that are convex combinations of the value attributions $\lambda_{i}$, such that the most general state can be written as a mixture $\sum_{i=0}^{\left(2^{n}\right)-1} p_{i} \lambda_{i}$, where $\sum_{i=0}^{\left(2^{n}\right)-1} p_{i}=1$ and $n$ denotes the number of observables.

The dynamics of this model now is such that after every measurement, the system may randomly execute a transition to a different state. Note that this transition does not depend on which measurement was carried out. This models the effect of noise introduced during measurement, i.e., after a noisy interaction with the system, further measurements will in general yield different results. We will now show that this is equivalent to the introduction of compatibility violations in a realistic experiment, and, crucially, that these violations may lead to false positives in Kochen-Specker tests.

Consider the evolution depicted in Fig. 1: a measurement of the observable $A$ is made on a system in the state $\lambda_{1}$, consequently producing the result +1 . Subsequently, the observable $B$ is measured, yielding -1 . Then, the system undergoes a state transition to $\lambda_{3}$, and a subsequent measurement of $A$ yields -1 . Thus, compatibility is violated.

Of course, this model cannot suffice to capture all 
quantum mechanical effects; in particular, for a priori incompatible observables, it is easy to show that its behavior differs from that of quantum mechanics: take a measurement sequence such as $A A A$. Without disturbances, both quantum mechanics and the model predict that the same result will be repeated three times; allowing for noise influences, there will be a small probability of disagreement. Measuring $A C A$, however, since $A$ and $C$ are not compatible, quantum mechanics predicts that the result for the second measurement of $A$ must be random, while in our model, it will agree with the first result up to possible probabilistic state changes (i.e., in our model, the probability distribution from which the value of $A$ is drawn will not differ whether it is the third measurement in the sequence $A A A$ or in the sequence $A C A$ ). However, Kochen-Specker tests are always carried out within compatible sets of observables, and, since we are (for the moment) only investigating what can be concluded from such a test alone, this is not our concern here. Our main point is that this simple model can invalidate some ideas to make Kochen-Specker tests robust against noise.

Let us now consider what happens during a measurement of the left-hand side of Eq. (1) if violations of compatibility are present. Then, if we denote by $A_{i}$ the observed value of $A$, given that the hidden variable state is $\lambda_{i},\left\langle\chi_{\mathrm{CHSH}}\right\rangle$ can be calculated as follows:

$$
\begin{aligned}
\left\langle\chi_{\mathrm{CHSH}}\right\rangle & =\sum_{i, j}\left(A_{i} B_{j}+B_{i} C_{j}+C_{i} D_{j}-D_{i} A_{j}\right) p_{i j} \\
& \equiv \sum_{i, j} K_{i j} p_{i j},
\end{aligned}
$$

where the $p_{i j}$ denote the probability that the evolution of the system is $\lambda_{i} \rightarrow \lambda_{j}$, that is, that the state during the first measurement was $\lambda_{i}$, which transitioned to $\lambda_{j}$ before the second one, and we have introduced the quantity $K_{i j}=A_{i} B_{j}+B_{i} C_{j}+C_{i} D_{j}-D_{i} A_{j}$. The maximum $K^{\max }$ of the coefficients $K_{i j}$ provides the upper bound

$$
\left\langle\chi_{\mathrm{CHSH}}\right\rangle=\sum_{i, j} K_{i j} p_{i j} \leq \max _{i j}\left\{K_{i j}\right\} \equiv K^{\max } .
$$

Each $K_{i j}$ represents the value of $\chi_{\mathrm{CHSH}}$, given the hidden variable evolution $\lambda_{i} \rightarrow \lambda_{j}$. It is easy to check that $K_{0,8}=4: \lambda_{0}=(++++)$ and $\lambda_{8}=(-+++)$, and thus, $\langle A B\rangle=\langle B C\rangle=\langle C D\rangle=+1$, while $\langle D A\rangle=-1$. Hence, a simple model that after each measurement changes the system's state from $\lambda_{0}$ to $\lambda_{8}$ will maximally violate the CHSH inequality; if the change happens only with a certain probability $p$, obviously any value between 2 and 4 can be achieved.

It should be noted that despite the evolution of the hidden variable, this model is noncontextual in the sense that whether or not a state transition is effected does not depend on the measured context. It thus seems surprising that this model can violate the CHSH inequality, apparently indicating contextual behavior. However, strictly speaking, noncontextuality simply does not apply in this case, as it is defined only under the assumption of perfect compatibility.

\section{CONNECTION WITH PREVIOUS WORKS}

An approach to rein in the effects of compatibility violations was proposed in Ref. [13]. There, several classes of error terms were proposed, such that additional measurements may be performed in order to quantify the degree of failure of a priori compatible observables to be compatible in the actual experiment, i.e., the degree of influence a measurement of $A$ has on the compatible measurement $B$, for example. We will concentrate, for the moment, on the first class of error terms from Ref. [13], which are those that have been experimentally implemented.

Based on an assumption of noise cumulation, that is, an assumption that additional measurements always lead to additional noise and thus a worse violation of compatibility, the inequality

$$
\begin{array}{r}
\left\langle\chi_{\mathrm{CHSH}}\right\rangle-p^{\text {err }}[B A B]-p^{\text {err }}[C B C] \\
-p^{\text {err }}[D C D]-p^{\operatorname{err}}[A D A] \leq 2,
\end{array}
$$

holds [13]. Here for instance $p^{\operatorname{err}}[B A B]$ is the probability that the second measurement of $B$ in the sequence $B A B$ disagrees with the first one.

However, it is clear that the model we discuss does not obey the assumption of cumulative noise: for an evolution such as $\lambda_{0} \rightarrow \lambda_{4} \rightarrow \lambda_{0}$, clearly both measurements of $B$ in the sequence $B A B$ agree, but if $B$ were measured in the second place of the sequence, then it would have yielded a value opposite to the first. Thus, the model is not necessarily constrained by Inequality (4); and in fact, since the error terms all vanish for such an evolution, it is clear that the model can violate it.

Alternatively, it may be noted that while the original CHSH-inequality is only concerned with measurement sequences of length 2 , the error terms contain only sequences of length 3, and thus, can only provide information about the system's behavior during such sequences. This criticism holds for the other two classes of error terms in Ref. [13] as well.

\section{MODIFIED INEQUALITIES}

However, another approach, which does not need any additional measurements or further assumptions, is possible. This amounts to essentially applying the ideas of Leggett and Garg to contextuality inequalities. Rather than employing the original inequalities proposed in Ref. [5], it is convenient for our purposes to use a slightly different formulation. Consider two different measurements $C$ and $C^{\prime}$, performed at two points in time. Then, $C\left(C+C^{\prime}\right)+C^{\prime}\left(C-C^{\prime}\right)= \pm 2$, and thus 


$$
\begin{array}{|lll|}
A=\sigma_{z} \otimes \mathbb{1} & B=\mathbb{1} \otimes \sigma_{z} & C=\sigma_{z} \otimes \sigma_{z} \\
a=\mathbb{1} \otimes \sigma_{x} & b=\sigma_{x} \otimes \mathbb{1} & c=\sigma_{x} \otimes \sigma_{x} \\
\alpha=\sigma_{z} \otimes \sigma_{x} & \beta=\sigma_{x} \otimes \sigma_{z} & \gamma=\sigma_{y} \otimes \sigma_{y}
\end{array}
$$

TABLE I: The Peres-Mermin square, with the Pauli matrices $\sigma_{i}$, and the $2 \times 2$ identity matrix $\mathbb{1}$. The observables in all rows and columns commute, and the product of all rows and the first two columns is equal to $\mathbb{1}$, while for the last column, $C c \gamma=-\mathbb{1}$.

$\left\langle C^{\prime} C\right\rangle+\langle C C\rangle+\left\langle C C^{\prime}\right\rangle-\left\langle C^{\prime} C^{\prime}\right\rangle \leq 2$, where $\left\langle C C^{\prime}\right\rangle$ denotes the correlation between $C$, measured at $t_{1}$, and $C^{\prime}$, measured at $t_{2}$.

We can now impose a similar time-ordering of observables on Eq. (1), to get

$$
\left\langle\chi_{\mathrm{CHSH}}\right\rangle=\langle A B\rangle+\langle C B\rangle+\langle C D\rangle-\langle A D\rangle \leq 2 .
$$

It is not hard to see that for Eq. (5), $K_{i j} \leq 2$ for all $(i, j)$ : if the first three terms are equal to +1 , the fourth is necessarily equal to +1 , as well. Hence, our model cannot violate Inequality (5), despite the violation of compatibility. Since in the case of a trivial evolution of the hidden variables, i.e. an evolution that leaves the state invariant, we recover the usual notion of (sequential) noncontextuality, an experimental test of Eq. (5) constitutes a test of quantum contextuality robust against the compatibility loophole.

It should be noted that the CHSH-inequality is not the only one that can be modified to hold in the case of compatibility violations: another important inequality proposed in Ref. [9] is based on the Peres-Mermin square ([19]; see Table I). Using the same reasoning as in the CHSH-case, the inequality

$$
\begin{aligned}
\left\langle\chi_{\mathrm{PM}}\right\rangle & =\langle A B C\rangle+\langle c a b\rangle+\langle\beta \gamma \alpha\rangle \\
& +\langle A a \alpha\rangle+\langle\beta B b\rangle-\langle c \gamma C\rangle \leq 4
\end{aligned}
$$

holds also in the case of imperfect compatibility between observables. In quantum mechanics, a value of $\left\langle\chi_{\mathrm{PM}}\right\rangle=6$ can be reached. Again, it is here the ordering of the measurement sequences that matters: the original inequality proposed in Ref. [9] followed the ordering indicated in Table I; but in this form, it is not hard to see that the inequality can be violated easily by our model. Interestingly, the ordering proposed here is also useful if the Mermin-Peres inequality should be used for estimating the dimension of a quantum system [20].

The importance of this scenario is that this inequality is state-independent, that is, one does not require a special quantum state for the violation (as is the case for the CHSH-inequality). Furthermore, an experiment using sequential measurements on trapped ions already implemented this scenario by measuring the observables in Table I in all possible permutations [14]. This experiment focused on the violation of the inequality as originally proposed in Ref. [9], and using this data, the observed value for Eq. (6) is $\left\langle\chi_{\mathrm{PM}}\right\rangle=5.35(4)$.
Not every noncontextuality inequality can be modified this straightforwardly, though. For example, the Klyachko-Can-Binicioğlu-Shumovsky inequality [10]

$$
\begin{aligned}
\left\langle\chi_{\mathrm{KCBS}}\right\rangle= & \langle A B\rangle+\langle B C\rangle+\langle C D\rangle \\
& +\langle D E\rangle+\langle E A\rangle \stackrel{N C H V}{\geq}-3 \stackrel{Q M}{\geq} 5-4 \sqrt{5}
\end{aligned}
$$

which exhibits a quantum violation even for a single qutrit system, as demonstrated experimentally in Ref. [21], cannot be rearranged appropriately. Nevertheless, our approach can be generalized: the modified inequality

$$
\begin{gathered}
\langle A B\rangle+\langle C B\rangle+\langle C D\rangle+\langle E D\rangle+\langle E A\rangle-\langle A A\rangle \\
\stackrel{N C H V}{\geq}-4 \stackrel{Q M}{\geq} 4-4 \sqrt{5}
\end{gathered}
$$

holds for any noncontextual hidden-variable evolution. The reason for this is that it enforces the ordering conditions as in Eq. (5): to maximize the left hand side of Eq. (8), for instance $A_{i}$ must equal $E_{j}$, as must $A_{j}$; however, then $A_{i}=A_{j}$, and thus, $\langle A A\rangle=1$. This shows that even in the case of a single qutrit a Kochen-Specker test ruling out our model can be undertaken. However, one should note that due to this modification, the relative quantum violation shrinks, since the absolute violation stays the same, while the absolute value of the classical expectation increases. Finally, it should be noted that a similar inequality like Eq. (8) has already been used in Ref. [21] in order to compensate for the fact that in this setup the observable $A$ was implemented in two different ways.

In fact, a recently proposed state-independent inequality violated by a single qutrit system [22] can be treated in the same way. This inequality features 13 observables $\left\{A^{1}, \ldots, A^{13}\right\}$, and the form that yields the maximum quantum violation is $[23,24]$

$$
\sum_{i} \Gamma_{i}\left\langle A^{i}\right\rangle+\sum_{i j} \Gamma_{i j}\left\langle A^{i} A^{j}\right\rangle \leq 16
$$

where the coefficients are as follows: $\Gamma_{i}=1$ for $i \in$ $\{4,7,10, \ldots, 13\}, \Gamma_{i}=2$ for $i \in\{1,5,6,8,9\}$, and $\Gamma_{i}=3$ for $i \in\{2,3\} ; \Gamma_{i j}=-1$ for $(i, j) \in\{(1,2),(1,3),(1,4)$, $(1,7),(4,10),(8,10),(9,10),(5,11),(7,11),(9,11)$, $(6,12),(7,12),(8,12),(4,13),(5,13),(6,13)\}, \Gamma_{i j}=-2$ for $(i, j) \in\{(2,3),(2,5),(2,8),(3,6),(3,9),(5,8),(6,9)\}$, and $\Gamma_{i j}=0$ else. By checking all possible hidden variable evolutions one verifies that the modified inequality [25]

$$
\sum_{i} \Gamma_{i}\left\langle A^{i}\right\rangle+\sum_{i j} \Gamma_{i j}\left\langle A^{i} A^{j}\right\rangle+4 \sum_{i}\left\langle A^{i} A^{i}\right\rangle \leq 68
$$

cannot be violated by noncontextually evolving models. However, since the maximum quantum value in this case is only $69+\frac{1}{3}$, the relative violation is reduced to $\frac{1}{51} \approx$ $1.96 \%$, compared to originally $\frac{1}{12} \approx 8.3 \%$. 


\section{CONCLUSION}

We have provided a novel approach to the compatibility problem in Kochen-Specker experiments. Using the idea of time-ordering, as first proposed by Leggett and Garg, we have derived new inequalities violated by quantum mechanics even in the case of imperfectly compatible measurements. This shows that with a careful ordering of the measurements classical models can be ruled out, which cannot be excluded with existing approaches [13]. Nevertheless, we are not claiming that our modified in- equalities allow a test of the Kochen-Specker theorem free from the compatibility loophole. Our results, however, show that with a simple reordering of the measurements a significantly larger class of hidden-variable models can be ruled out.

We thank C. Roos for providing us with the data from their experiment, and C. Budroni and D. Bruß for valuable discussions. This work has been supported by the EU (Marie Curie CIG 293993/ENFOQI) and BMBF (projects QuOReP and QUASAR).
[1] J.S. Bell, Physics 1, 195 (1964).

[2] A. Einstein, B. Podolsky, N. Rosen, Physical Review 41, 777 (1935).

[3] A. Aspect, P. Grangier, G. Roger, Phys. Rev. Lett. 47, 460 (1981); A. Aspect, P. Grangier, G. Roger, Phys. Rev. Lett. 49, 91 (1982); A. Aspect, J. Dalibard, G. Roger, Phys. Rev. Lett. 49, 1804 (1982).

[4] G. Weihs, T. Jennewein, C. Simon, H. Weinfurter, A. Zeilinger, Phys. Rev. Lett. 81, 5039 (1998).

[5] A.J. Leggett, A. Garg, Phys. Rev. Lett. 54, 857 (1985).

[6] S. Kochen, E. P. Specker, J. Math. Mech. 17, 59 (1967).

[7] J. S. Bell, Rev. Mod. Phys. 38, 447 (1966).

[8] N. D. Mermin, Rev. Mod. Phys. 65, 803 (1993).

[9] A. Cabello, Phys. Rev. Lett. 101, 210401 (2008).

[10] A. A. Klyachko, M. A. Can, S. Binicioğlu, A. S. Shumovsky, Phys. Rev. Lett. 101, 020403 (2008).

[11] D. A. Meyer, Phys. Rev. Lett. 83, 3751 (1999).

[12] A. Kent, Phys. Rev. Lett. 83, 3755 (1999).

[13] O. Gühne, M. Kleinmann, A. Cabello, J-Å. Larsson, G Kirchmair, F. Zähringer, R. Gerritsma, C. F. Roos, Phys. Rev. A 81, 022121 (2010).

[14] G. Kirchmair, F. Zähringer, R. Gerritsma, M. Kleinmann, O. Gühne, A. Cabello, R. Blatt, C. F. Roos, Nature 460, 494 (2009).

[15] M. Wilde, A. Mizel, Found. Phys. 42, 256 (2012).
[16] A. Cabello, M. Terra Cunha, Phys. Rev. Lett. 106, 190401 (2011)

[17] J. F. Clauser, M. A. Horne, A. Shimony, and R. A. Holt, Phys. Rev. Lett. 23, 880 (1969).

[18] B. S. Tsirelson, Lett. Math. Phys. 4, 93 (1980).

[19] A. Peres, Phys. Lett. A 151, 107 (1990); N. D. Mermin, Phys. Rev. Lett. 65, 3373 (1990).

[20] O. Gühne, C. Budroni, A. Cabello, M. Kleinmann, and J.-Å. Larsson, arXiv:1302.2266.

[21] R. Lapkiewicz, P. Li, C. Schaeff, N.K. Langford, S. Ramelow, M. Wieśniak, and A. Zeilinger, Nature (London) 474, 490 (2011).

[22] S. Yu, C. H. Oh, Phys. Rev. Lett 108, 030402 (2012).

[23] M. Kleinmann, C. Budroni, J.-Å. Larsson, O. Gühne, A. Cabello, Phys. Rev. Lett. 109, 250402 (2012).

[24] X. Zhang, M. Um, J. Zhang, S. An, Y. Wang, D.-l. Deng, C. Shen, L. Duan and K. Kim, Phys. Rev. Lett. 110, 070401 (2013).

[25] There is no ordering ambiguity for the single measurements $\left\langle A^{i}\right\rangle$ since we consider disturbances to result from measurement interactions; a single measurement can always be considered to occur in the first position of a measurement sequence. 